\title{
Efficacy and Safety of Actively Personalized Neoantigen Vaccination in the Management of Newly Diagnosed Glioblastoma: A Systematic Review
}

\author{
Muhammad Khan (1D ${ }^{1,2}$ \\ Xianming $\mathrm{Li}^{\mathrm{l}}$ \\ Maosheng Yan' \\ Zihuang $\mathrm{Li}^{\prime}$ \\ Hongli Yang' \\ Guixiang Liao (D) \\ 'Department of Oncology, Shenzhen \\ People's Hospital, The First Affiliated \\ Hospital of Southern University of \\ Science and Technology, Shenzhen, \\ 518020, People's Republic of China; \\ ${ }^{2}$ Department of Oncology, The First \\ Affiliated Hospital of Anhui Medical \\ University, Hefei, Anhui Province, \\ People's Republic of China
}

Correspondence: Guixiang Liao Department of Oncology, Shenzhen People's Hospital, The First Affiliated Hospital of Southern University of Science and Technology, Shenzhen, 5 I8020, People's Republic of China Tel +86 7552294240 I

Email liaoguixiang@163.com
Purpose: Glioblastoma (GBM) shows frequent relapse and is highly resistant to treatment; therefore, it is considered fatal. Various vaccination protocols that have been tested in patients with GBM, which is the most common and aggressive primary brain tumor, have indicated safety and efficacy, to some extent, when used alone or in combination with standard of care. Recently, neoantigen-based personalized vaccines have shown tremendous immunogenicity and safety in GBM. We aimed to systematically review the medical literature for clinical trials to evaluate the efficacy and safety of neoantigen-based personalized vaccines for newly diagnosed GBM.

Methods: We conducted a literature search for clinical trials on PubMed, Cochrane Library, China National Knowledge Infrastructure, and ClinicalTrials.gov until March 20, 2021. The primary outcomes of interest were immunogenicity and safety of the therapy. Efficacy outcomes, such as progression-free survival and overall survival, were secondary outcomes of interest.

Results: Two clinical trials involving 24 patients were included in this review. High immunogenicity was observed in both studies. The GAPVAC-101 trial reported 50\% APVAC1-induced and 84.7\% APVAC2-induced immunogenicity with CD8+ and CD4+ $\mathrm{T}$ cell responses in 92\% (12/13) and 80\% (8/10) immune responders, respectively. Two out of five patients showed CD4+ and CD8 $+\mathrm{T}$ cell responses in the study by Keskin et al. Dexamethasone use had limited immunogenicity in a trial by Keskin et al (6/8). No serious treatment-related adverse events were reported.

Conclusion: Actively personalized vaccines aimed at unmutated peptides and neoantigens for patients with GBM are safe and highly immunogenic, particularly when administered in combination. Larger studies are warranted to investigate the role.

Keywords: glioblastoma, GBM, active immunotherapy, personalized peptide vaccination, neoantigen, immunogenicity, safety

\section{Introduction}

Glioblastoma multiforme (GBM) accounts for $14.9 \%$ of primary brain tumors and $55.4 \%$ of all gliomas, but it is the most common (46.6\%) of all malignant tumors of the central nervous system. Its incidence rate is 3.20 per 100,000 population. According to the CBTRUS Statistical Report 2009-2013, GBM is significantly prevalent in males compared to that in females and in whites compared to that in blacks in the United States. ${ }^{1}$ GBM exists in the primary and secondary forms. Primary GBM, which represents the majority of the tumors $(90 \%)$, develops de 
novo and has no lower grade precursor malignancy. Secondary GBM is a grade IV glioma and has low-grade diffuse astrocytoma (grade II) or anaplastic astrocytoma (grade III) as its precursor. ${ }^{2}$ Clinical manifestations of GBM include physical, neurological, and psychological signs and symptoms, such as headache, nausea and vomiting, visual and language disturbances, motor weakness, cognitive impairment, memory loss, and personality changes. $^{3}$

GBM is considered fatal because it frequently relapses and is highly resistant to therapy. ${ }^{4}$ Patients with GBM who have not received treatment reported a median survival time of only 3 months. ${ }^{5}$ Standard of care (SOC), which consists of surgical resection, temozolomide chemotherapy, and radiotherapy, has improved the median survival time to $12-18$ months. ${ }^{5,6}$ Targeted therapy involving the addition of bevacizumab and everolimus to the SOC has comparatively improved outcomes. ${ }^{7,8}$ Recent advances in immunotherapy, especially immune checkpoint inhibitors (ICIs), have shown promise for several cancers. However, monotherapy with ICIs has failed to improve outcomes in patients with GBM. ${ }^{9}$ Hence, GBM is also termed as a "cold tumor." However, several forms of vaccinations have been administered for GBM, which have shown a slight surge in progression-free survival (PFS) and overall survival (OS) in these patients. ${ }^{10}$

Three main types of antigens that are targeted in GBM vaccinations are being tested clinically. They include tumorassociated antigens (TAAs), tumor-specific antigens (TSAs), and tumor lysate. ${ }^{11}$ Neoantigens are TSAs resulting from somatic DNA alterations in the form of nonsynonymous point mutations, insertions or deletions, gene fusions, and frameshift mutations. Most recently, there has been a boom in the application and success of neoantigen-based vaccination for melanoma. ${ }^{12}$ Moreover, in other cancers, such as melanoma, colorectal cancer, and non-small cell lung cancer (NSCLC), somatic mutation burden was correlated with increased survival and clinical benefit derived from ICI application. ${ }^{13-17}$ This vaccine represents a more personalized form of vaccine, as it accounts for cancer-to-cancer variation for specific cancer types as well as patient-topatient variations. Recently, two trials have assessed neoantigen-based peptide vaccines for newly diagnosed patients with GBM. Both these trials have reported tremendous CD8 + and $\mathrm{CD} 4+\mathrm{T}$ cell responses against the tumor and tumor infiltration of these cells, making them "hot tumors." ${ }^{\text {"18,19 } \mathrm{We}}$ aimed to conduct a systematic review of studies to evaluate the efficacy and safety of neoantigen-based vaccines for GBM, keeping in mind the future prospects.

\section{Materials and Methods}

Preferred Reporting Items for Systematic Reviews and Meta-Analyses (PRISMA) guidelines were followed for reporting. ${ }^{20}$ A protocol of this study is registered on PROSPERO: CRD42021248719.

\section{Inclusion Criteria}

\section{Patients and Study Types}

Patients with GBM who received personalized neoantigen vaccines were included. Only clinical trials (CTs) were included in this study. Retrospective studies, case reports, and/or commentary were excluded.

\section{Types of Interventions}

The intervention was personalized neoantigen-based vaccine for patients with GBM.

\section{Outcomes of Interest}

Immunogenicity and safety were the primary outcomes of interest. The secondary outcomes of interest included PFS and OS.

\section{Search Strategy \\ Databases}

We conducted a literature search in PubMed, Cochrane Library, China National Knowledge Infrastructure (CNKI), and ClinicalTrials.gov until August 20, 2019. "English only" language restriction was applied. Furthermore, references of relevant studies were searched for identifying more studies.

\section{Study Selection}

The selected studies were imported into Endnote X9 software for organizing, screening, and removing duplicates. After removal of duplicates, the titles and abstracts of the studies were screened. Studies that met the exclusion criteria were excluded. Study selection was performed by two independent reviewers. Full text and supplementary materials were obtained for the selected studies. Any disagreements were resolved by discussion among the authors' team.

\section{Data Extraction}

The Cochrane Collaboration Data Collection form-randomized controlled trials (RCTs) and non-RCTs-was used and modified for data extraction. We collected information on attributes of the studies, study design, first 
author, country of research, publication year, number of participants, and characteristics of vaccine development and delivery. Characteristics of patients, such as age, Karnofsky Performance Scale (KPS), human leukocyte antigen (HLA) allotypes, MGMT methylation status, and median number of vaccinations. Finally, data of the outcomes of interest were extracted, which included data on immunogenicity and safety and patient survival.

\section{Assessment of Risk of Bias}

Risk of bias was assessed using the Cochrane tools. ${ }^{21}$

\section{Measurement of Treatment Effect and Data Synthesis}

The extracted data were incorporated into the table form. Immunogenicity was recorded as the number of immunogenic peptides, observed $\mathrm{CD} 8+$ and $\mathrm{CD} 4+\mathrm{T}$ cell responses, and tumor infiltration of $\mathrm{T}$ cells.

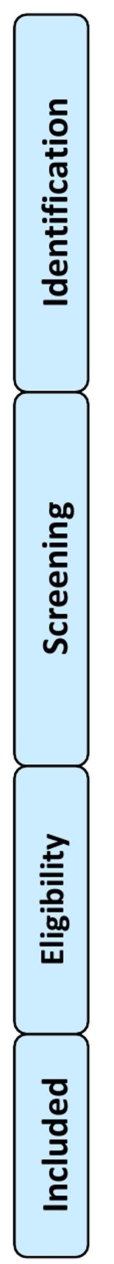

Records identified through database searching $(n=4902)$

\section{Results}

\section{Studies' and Patients' Characteristics}

Two CTs involving 24 patients were included in this systematic review (Figure 1). ${ }^{18,19}$ All patients had newly diagnosed GBM and had received surgery, radiotherapy, or chemoradiotherapy, followed by personalized neoantigenbased vaccine. GAPVAC-101 patients $(n=16)$ received two synthesized peptide vaccines, one aimed at unmutated peptides (APVAC1) and the other aimed at neoantigens (APVAC2). APVAC1 was formulated using a preconstructed library of HLA-presented non-mutated antigens in patients with GBM. APVAC1 consisted of seven best-ranked class I peptides plus two class II (pan-DR antigen) and a viral peptide. The participants $(n=8)$ in Keskin et al's study received only a neoantigen-based vaccine formulation (NeoVax). One case study that reported neoantigen-specific $\mathrm{T}$ cell responses in a patient
Additional records identified through other sources $(n=3)$

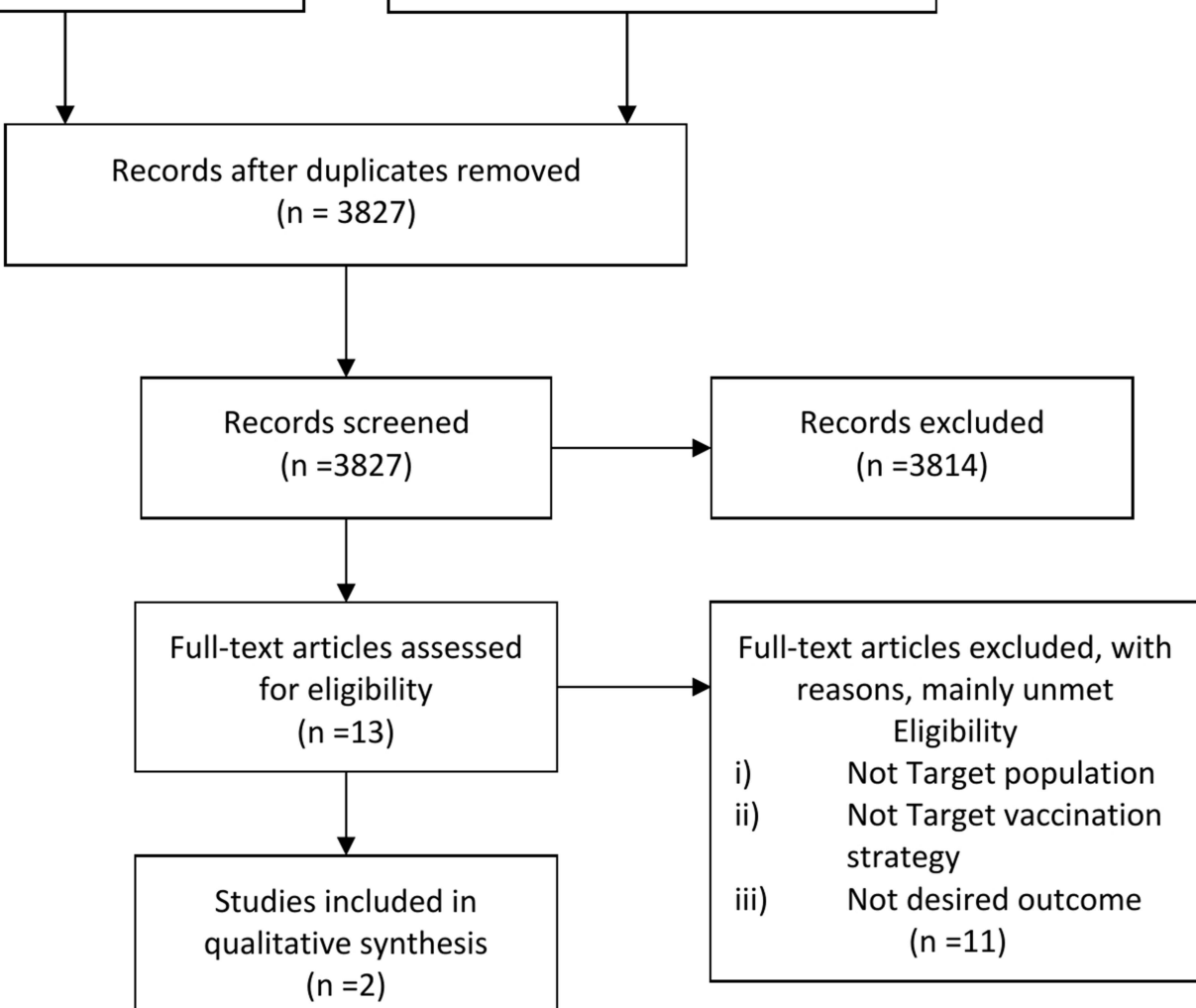

Figure I PRISMA flow diagram.

Notes: PRISMA figure adapted from Moher D, Liberati A, Altman D, Tetzlaff J et al. The PRISMA statement for reporting systematic reviews and meta-analyses of studies that evaluate health-care interventions: explanation and elaboration. Journal of clinical epidemiology. 2009; 62(I0). Creative Commons ${ }^{20}$. 
with GBM after the administration of neoantigen vaccine was excluded. $^{22}$ The general characteristics of the studies, participants, and vaccines are listed in Table 1.

\section{Immunogenicity}

Three vaccines (APVAC1, APVAC2, and NeoVax) were applied: 2 (APVAC1 and APVAC2) in the GAPVAC-101 trial and 1 (NeoVax) by Keskin et al. APVAC1 produced $50 \%$ immunogenicity, and APVAC2 induced $84.7 \%$ immunogenicity. APVAC1 produced CD8+ $\mathrm{T}$ cell responses of the central memory type, and APVAC2, which was aimed at neoantigens, produced primarily CD4+ T cell responses. In the study by Keskin et al, two of the five patients who received at least one boost after priming but did not receive dexamethasone for side effects at priming showed immunogenicity. Keskin et al also revealed that both kinds of responses of $\mathrm{CD} 4+\mathrm{T}$ cells and CD8+ $\mathrm{T}$ cells enriched in the memory phenotype (Table 2).

\section{APVACI}

A total of 13 patients received 87 APVAC1 peptides. Eleven of the 13 patients showed immunogenicity with sustained immune responses of central memory CD8+ $\mathrm{T}$ cells. Forty-five of the 87 vaccination peptides were immunogenic, revealing a $51.7 \%$ immunogenicity. Each APVAC1 had two peptides directed at class II antigens (pan-DR antigens). Overall, 13 patients had received 26 peptides, of which 9 showed immunogenicity to one or both unmutated pan-DR antigens. Thirteen of the 26 peptides administered were immunogenic, revealing 50\% immunogenicity. These peptides mainly induced CD4+ $\mathrm{T}$ cell responses.

\section{APVAC2}

Overall, ten patients were evaluated for APVAC2 immunogenicity. Eight patients (80\%) demonstrated neoepitopespecific immune responses, predominantly CD4+ T cell responses. Eleven mutated APVAC2 peptides induced isolated CD4+ $\mathrm{T}$ cell responses or $\mathrm{CD} 4+$ plus $\mathrm{CD} 8+\mathrm{T}$ cell responses of the 13 vaccinated individuals, showing an $84.7 \%$ immunogenicity. The CD4+ $\mathrm{T}$ cell responses were predominantly of the $\mathrm{T}_{\mathrm{H}} 1$ phenotype and were multifunctional. None of these mutated APVAC2 peptides had induced isolated CD8+ T-cell responses. APVAC2 unmutated peptides ${ }^{6}$ induced $\mathrm{CD} 8+\mathrm{T}$ cell responses only once (Patient 8).

\section{NeoVax}

Overall, two patients who did not receive dexamethasone showed immunogenic responses. Patient 7 primarily responded to pool $\mathrm{C}$ peptides with $\mathrm{CD} 4+\mathrm{T}$ cell responses, primarily against the mutated neoepitopes. Patient 8 responded to two pools (pools A and B) with CD4+ T cell responses against three neoepitopes. Two mutated neoepitopes were targeted preferentially over the wild type, whereas 1 (COX18) neoepitope showed similar reactivity between mutant and wild type. Approximately $20-30 \%$ of the $\mathrm{CD} 4+$ and $\mathrm{CD} 8+\mathrm{T}$ cell responses were polyfunctional, and half of these expressed at least one effector cytokine.

\section{Tumor Infiltration of T Cells}

Both studies revealed tumor infiltration of $\mathrm{CD} 8+$ and $\mathrm{CD} 4+$ $\mathrm{T}$ cells. GAPVAC-101 reported that a single patient (Patient 8) had tumor resection following recurrence at 26.8 months after diagnosis, demonstrating high infiltration of $\mathrm{T}$ cells and a favorable CD8+ T/FOXP3+ Treg cell ratio. Keskin et al reported five patients (Patients 3, 4, 5, 7, and 8) with disease progression (PFS; median=17.3 weeks; range, 6.7-26.3) underwent surgery after vaccination. Two patients (Patients 7 and 8) showed a significant increase in CD8+ $\mathrm{T}$ cell infiltration into the tumor at relapse compared to that at baseline $(\mathrm{p}=0.006)$. Compared to Patients 3, 4, and 5 who received dexamethasone, Patients 7 and 8 demonstrated an increase in $\mathrm{CD} 8+(\mathrm{p}=0.02)$ and $\mathrm{CD} 4+\mathrm{T}$ cells $(\mathrm{p}=0.008)$.

\section{Safety and Tolerability}

Both studies reported treatment-related adverse events. Injection site disorders were the prominent side effects, particularly in the GAPVAC-101 study. Other events were mild (Table 3).

\section{Progression-Free Survival and Overall Survival}

Both studies reported PFS for all participants. Keskin et al revealed a median PFS of 7.6 months $(n=8)$. The GAPVAC-101 study reported a median PFS of 14.2 months $(\mathrm{n}=15)$. A median OS of 29 months was reported in the GAPVAC-101 study, whereas Keskin et al reported a median OS of 16.8 months.

\section{Checkpoint Inhibition Compatibility}

ICIs when administered as monotherapy have failed in GBM treatment. ${ }^{9}$ However, it is anticipated to be an adjuvant with personalized neoantigen-based vaccine, as 
Table I General Characteristics of the Studies and Patients

\begin{tabular}{|c|c|c|c|}
\hline Clinical Trials & \multirow[t]{2}{*}{ Keskin et al ${ }^{19}$} & \multirow[t]{2}{*}{ GAPVAC- $10 \mathrm{I}^{18}$} & \multirow[t]{2}{*}{ Total } \\
\hline Characteristics & & & \\
\hline $\begin{array}{l}\text { Number of participants } \\
\text { Age (median; years) } \\
\text { Female }\end{array}$ & $\begin{array}{c}\mathrm{N}=8(100) \\
65 \text { (range, } 45-73) \\
6(75)\end{array}$ & $\begin{array}{c}\mathrm{N}=16(100) \\
52.5 \text { (range, } 25-70) \\
7(44)\end{array}$ & $\begin{array}{l}24(100) \\
13(54)\end{array}$ \\
\hline \multicolumn{4}{|l|}{ KPS } \\
\hline $\begin{array}{l}100 \\
90 \\
80 \\
70\end{array}$ & $\begin{array}{l}0 \\
6(75) \\
I(13) \\
I(13)\end{array}$ & $\begin{array}{c}4(25) \\
6(37.5) \\
5(31.25) \\
1(6.25)\end{array}$ & $\begin{array}{l}4(16.6) \\
12(50) \\
6(25) \\
2(8.3)\end{array}$ \\
\hline MGMT methylation & MGMT unmethylated & $28.6 \%$ MGMT hypermethylated & \\
\hline IDHI wild-type & $8(100)$ & - & \\
\hline Dexamethasone use & $6(75)$ & 1 & $7(29)$ \\
\hline $\begin{array}{l}\text { Mutations per tumour } \\
\text { (median; range) }\end{array}$ & 59 (32-93) Coding mutations per tumour & $\begin{array}{c}36(19-84) \text { somatic, non-synonymous } \\
\text { mutations }\end{array}$ & \\
\hline $\begin{array}{l}\text { Surgery to Ist neovax } \\
\text { (median weeks; range) }\end{array}$ & $18.6(17.1-25.0)$ & & \\
\hline Vaccine composition & $\begin{array}{c}\text { NeoVax: } 7-20 \text { peptides ( } 15-30 \text { aa) divided into pools of } \\
\text { 3-5 peptides (9-10aa) designated as A, B, C, D }\end{array}$ & $\begin{array}{l}\text { APVACI: } 7 \text { class I peptides }+2 \text { class II } \\
\text { peptides }+ \text { a viral marker peptide } \\
\text { APVAC2: } 20 \text { de novo synthesized } \\
\text { peptides ( } 14 \text { mutated and } 6 \text { unmutated) }\end{array}$ & \\
\hline Adjuvant & Admixed with poly-ICLC & $\begin{array}{l}\text { GM-CSF (intra- dermal injection) and } \\
\text { poly-ICLC (subcutaneous injection) }\end{array}$ & \\
\hline $\begin{array}{l}\text { Vaccination peptides/ } \\
\text { patient (median; range) }\end{array}$ & $12(7-20)$ & APVACI/2: $12 / 10$ & \\
\hline HLA-restriction & None & $\begin{array}{l}\text { HLA-A*02:01 } \\
\text { HLA-A*24:02 }\end{array}$ & \\
\hline
\end{tabular}

Note: All data given as frequencies and (percentages) unless otherwise indicated.

Abbreviations: N, number; MGMT, O[6]-methylguanine-DNA methyltransferase, IDHI= isocitrate dehydrogenase I; HLA, human leukocyte antigen; poly-ICLC, polyinosinic and polycytidylic acid, stabilized with poly-l-lysine and carboxymethylcellulose; GM-CSF, granulocyte-macrophage colony-stimulating factor; HLA-A*02/ HLA-A*24, human leukocyte antigen serotype determined by the antibody recognition of the $\alpha 2$ domain $/ \alpha^{24}$ subset of the HLA-A $\alpha$-chain.

the new $\mathrm{T}$ cell responses disappear owing to $\mathrm{T}$ cell

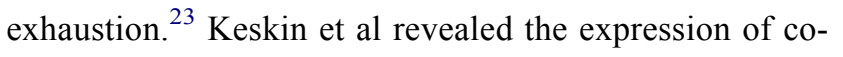
inhibitory molecules, such as TIM-3, TIGIT, PD-1, CTLA-4, and LAG-3 in combinations of 2 or 3 on $\mathrm{T}$ cells, including both $\mathrm{CD} 4+$ and $\mathrm{CD} 8+\mathrm{T}$ cells. A subset of CD8+ tumor-infiltrating T cells were positive for PD-1 post-vaccination, and their levels increased significantly with vaccination in Patients 7 and $8(\mathrm{p}=0.04)$. GAPVAC-101 also revealed a mild to moderate increase in PD-1 expression on CD8+ T cells to APVAC1 $(n=16)$ and APVAC2 (Patient 14) post-vaccination.

\section{Dexamethasone Effect}

Dexamethasone, as required for treating brain edema in patients undergoing chemoradiotherapy, has been shown to cause immunosuppression by impairment of $\mathrm{T}$ cell proliferation (CD4+ and CD8 $+\mathrm{T}$ cells). ${ }^{24}$ Keskin et al pointed out the use of dexamethasone as the cause of immune unresponsiveness, as patients $(n=6)$ who had required it for treating side effects were unable to show immunogenicity. One patient in the GAPVAC-101 study (Patient 9) also required high-dose dexamethasone and was not evaluable for immunogenicity. 
Table 2 Immunogenicity of Personalized Unmutated \& Neoantigen Vaccination

\begin{tabular}{|c|c|c|c|c|c|}
\hline Vaccines & Peptides & Number & Immunogenic & Patients & T Cells Responses \\
\hline \multirow{2yyyyn}{*}{ APVACI } & Class I & 87 & $45(51.7 \%)$ & $12 / 13(92.3 \%)$ & CD8+ T cells \\
\cline { 2 - 5 } & Class II & 26 & $13(50 \%)$ & $9 / 13(69.2 \%)$ & CD4+ T cells \\
\cline { 2 - 5 } APVAC2 & Mutated & 13 & $11(84.7 \%)$ & $8 / 10(80 \%)$ & CD4+ and CD4+ plus CD8+ T cells \\
\cline { 2 - 4 } NEOVAX & $\begin{array}{c}7-20 \text { peptides (I5-30aa) divided into } \\
\text { pools of 3-5 peptides (9-10aa) } \\
\text { designated as A, B, C, D }\end{array}$ & 60 & $\begin{array}{c}\text { Pool C peptides } \\
\text { (pt-7) A, B pools } \\
\text { (pt-8) }\end{array}$ & $2 / 5$ & \multirow{2}{*}{$\begin{array}{c}\text { Mainly CD4+ T cells (Pt 7 and 8) and } \\
\text { lower frequencies of CD8+ T cells } \\
\text { (only Pt 7) }\end{array}$} \\
\hline
\end{tabular}

Table 3 Treatment Related Adverse Events

\begin{tabular}{|c|c|c|c|c|c|}
\hline \multirow[t]{2}{*}{ Adverse Event } & \multicolumn{2}{|c|}{ All TRAE } & \multirow{2}{*}{$\begin{array}{l}\text { Grade }>2 \\
\text { Keskin et al }\end{array}$} & \multirow{2}{*}{$\begin{array}{c}\text { Grade } \geq 3 \\
\text { GAPVAC- } 101^{18}\end{array}$} & \multirow[t]{2}{*}{ Total } \\
\hline & GAPVAC- $101^{18}$ & Keskin et al ${ }^{19}$ & & & \\
\hline Total events & 34 & 9 & 2 & 5 & 43 \\
\hline Chills & 2 & I & & & 3 \\
\hline Dizziness & & I & & & I \\
\hline Fatigue & 3 & 1 & I & & 4 \\
\hline Flushing & & I & & & I \\
\hline Headache & 2 & I & I & & 3 \\
\hline Myalgia & & 2 & & & 2 \\
\hline Nausea & 2 & 1 & & & 3 \\
\hline Injection site reaction & 14 & I & & I & 15 \\
\hline Influenza like illness & 3 & & & & 3 \\
\hline Leukopenia & 2 & & & 1 & 2 \\
\hline Anaphylactic reaction & 2 & & & 1 & 2 \\
\hline Rash & I & & & & I \\
\hline Lymphopenia & I & & & I & I \\
\hline Brain edema & 1 & & & 1 & I \\
\hline Skin odor abnormal & I & & & & I \\
\hline
\end{tabular}

Note: All data given as frequencies.

Abbreviation: TRAE, treatment-related adverse events.

\section{Discussion}

A familiar pattern of development is being observed in vaccination-based immunotherapy to overall advancements in cancer therapeutics - from generalized treatment to more personalized therapy. A series of vaccination regimens targeting three main categories of antigens in GBM, namely, TAAs, TSAs, and tumor lysate, were investigated in several trials, which revealed comparative safety and better efficacy. ${ }^{11}$ These vaccine protocols included Rindopepimut targeting EGFRvIII (a mutant form of epidermal growth factor receptor present in $20-30 \%$ of patients with GBM), ${ }^{25-28}$ WT-1 vaccine (Wilm's tumor gene 1) targeting 9-mer modified WT-1 peptide, ${ }^{29-32}$ SurVaxM targeting survivin (a glioma cell survival protein), ${ }^{33}$ and a prophage (G 100, G200, Vitespen, Oncophage) targeting the heat shock protein peptide 
complex (HSPPC-96). ${ }^{34-36}$ Rindopepimut alone showed superior PFS and/or OS over temozolomide in matched/ historical controls in two preliminary trials (ACTIVATE: $\mathrm{n}=18$; ACT II: $\mathrm{n}=22$ ) but failed to show any improvement in PFS or OS with the addition of temozolomide (ACT III and ACT IV). ${ }^{25-28}$ WT-1 vaccine trials mainly aimed at safety and clinical response evaluations. The results indicated that WT-1 was safe, with evidence of clinical and humoral responses. ${ }^{29-32}$ SurVaxM also induces immunogenicity with no safety concerns in recurrent GBM. ${ }^{33}$ Likewise, HSPPC-96 vaccine also proved its immunogenicity, with improvement in PFS (median 11 to 17.8 months) and OS (median 23.8 to 31.4 months). ${ }^{34-36}$ Gliovax, a vaccine made from autologous antigens of a patient's own tumor in combination with allogeneic antigens from other patients with GBM, revealed 100\% 6-month OS in a smaller study $(\mathrm{n}=9)$ involving recurrent GBM $(\mathrm{rGBM}){ }^{37}$ Dendritic cell-based vaccines pulsed with tumor autologous lysates or tumor-associated multiple epitopes have also been shown to be safe and efficacious in several Phase I and Phase II studies. ${ }^{38-44}$ IMA950, a more personalized form of vaccine, was tested using patient-associated antigens found on HLA antigen surface receptors and was also shown to be safe and efficacious. ${ }^{45}$ A Phase III study evaluating personalized peptide vaccine, the method that was applied in the GAPVAC-101 trial for unmutated antigen (APVAC1) selection, showed safety but no efficacy compared to the control. ${ }^{46}$ More or less, such vaccines alone or their integration into the SOC have shown safety and, to some extent, better efficacy than SOC alone only in smaller trials, as shown in Table 4.

Neoantigens represent a more personalized cancer treatment and patient-specific vaccination. This vaccination has already shown higher immunogenicity and efficacy in patients with melanoma, which carries a higher mutational burden. ${ }^{47,48}$ By contrast, GBM represents a less mutation-carrying tumor with low infiltration of intratumoral $\mathrm{T}$ cells. ${ }^{23}$ Therefore, the results of such high immunogenicity and efficacy, particularly in the GAPVAC-101 trial, show promise for this group of patients. HLArestricted personalized peptide vaccines (APVAC1) as well as neoantigen-containing peptides (APVAC2) showed $50 \%$ and $84.7 \%$ immunogenicity and, more importantly, $92 \%$ and $80 \%$ immune responders, respectively. This study also reported a median overall survival of 29 months, which is higher than that reported in a previous study (Table 4). However, this represents a combination of two vaccine strategies applied for the first time. The HLA- restricted personalized peptide vaccine strategy alone failed to demonstrate any efficacy in a phase III trial for patients with GBM. ${ }^{46}$ Similarly, Keskin et al also applied only neoantigen-based vaccines, which demonstrated immunogenicity in only two of the eight patients and an OS of 16.8 months. These studies included a small number of patients, which makes it difficult to analyze the efficacy outcome; nevertheless, a combined approach may provide a better option warranting further exploration in larger trials.

T cell exhaustion through inhibitory checkpoints, such as CTLA-4 (cytotoxic T lymphocyte-associated antigen-4), PD1 (programmed cell death-1), TIGIT (T cell immunoreceptor with Ig and ITIM domains), and TIM-3 (T cell immunoglobulin domain and mucin domain-3), reflects a postvaccination scenario for these patients. Both these trials revealed evidence for increased expression of PD-1 on CD8 $+\mathrm{T}$ cells (circulating and tumor-infiltrating $\mathrm{T}$ cells) postvaccination. ${ }^{18,19}$ Although ICIs when administered as monotherapy have failed in patients with GBM, exhausted CD8+ T cells in GBM could provide the rationale for administering ICIs in combination with personalized vaccine. Although two or three of the co-inhibitory receptors (CTLA-4, PD-1, TIGIT, and TIM-3) were expressed on these immune cells, double checkpoint inhibition of nivolumab and ipilimumab was not safe in patients with GBM. ${ }^{9}$ Somatic mutation load has been correlated with deriving clinical benefit from ICIs in multiple cancers, including NSCLC, melanoma, and colorectal cancers, as previously mentioned. ${ }^{14-17,49}$ However, in patients with GBM, the mutational/predicted-neoantigen burden was revealed as a biomarker of resistance in a study investigating preclinical efficacy and predictive biomarkers of responsiveness to ICIs. ${ }^{50}$ Dexamethasone also upregulates the expression of CTLA-4 and PD-1, but only CTLA-4 blockade hinders dexamethasone-induced immunosuppression. ${ }^{51}$ Hence, the addition of ICIs to SOC and vaccination may prolong the survival outcome of patients with GBM. Nonetheless, immune checkpoint inhibition in GBM is challenging and is under investigation with other SOC modalities. ${ }^{52}$

There were some inherent limitations that should be taken into account. Dexamethasone administration may have limited the trial by Keskin et al. Dexamethasone induces immunosuppression through depletion of lymphocytes (CD4+ and CD8+ $\mathrm{T}$ cells), impairment of $\mathrm{T}$ cell proliferation and differentiation, and increase of regulatory $\mathrm{T}$ cell proliferation and activation. ${ }^{24,46}$ Therefore, dexamethasone as an anti-inflammatory drug may have 


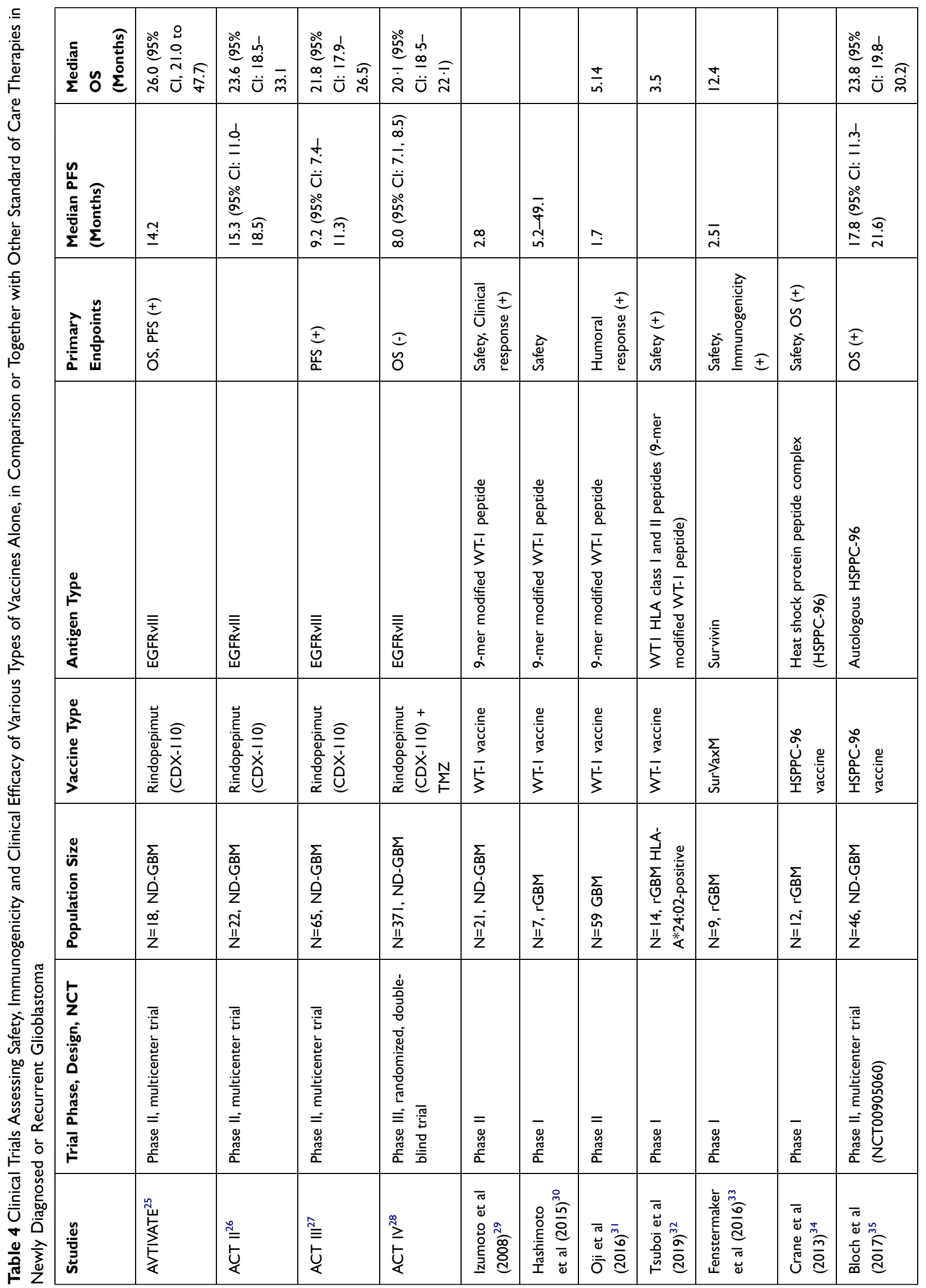




\begin{tabular}{|c|c|c|c|c|c|c|c|c|c|}
\hline 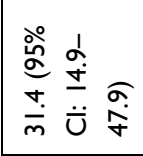 & ơ & $\stackrel{\infty}{\stackrel{\infty}{m}}$ & 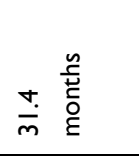 & 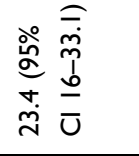 & 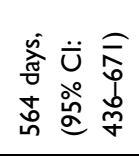 & 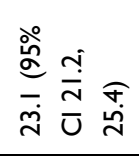 & 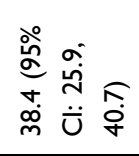 & 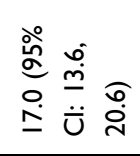 & $\underline{\tilde{m}}$ \\
\hline 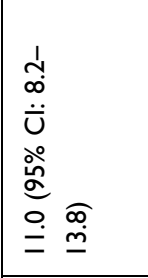 & 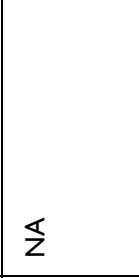 & $\stackrel{\sigma}{\underline{\alpha}}$ & $\underline{\dot{\rho}}$ & 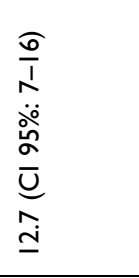 & 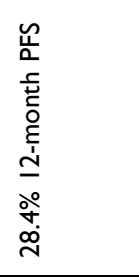 & $\mathbb{z}$ & 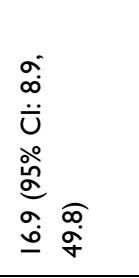 & 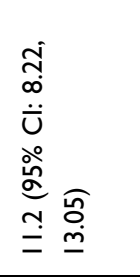 & 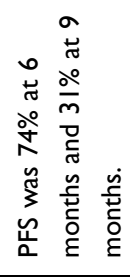 \\
\hline 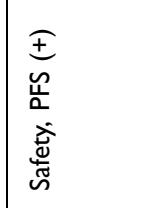 & 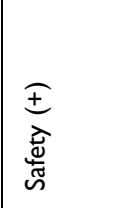 & & 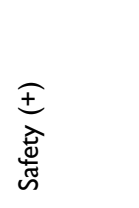 & $\underset{\frac{f}{5}}{\frac{f}{2}}$ & 竞 & $\underset{\frac{f}{2}}{\frac{f}{2}}$ & 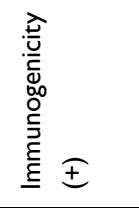 & $\begin{array}{l} \pm \\
\Xi \\
0\end{array}$ & 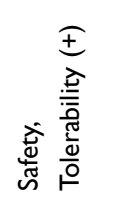 \\
\hline 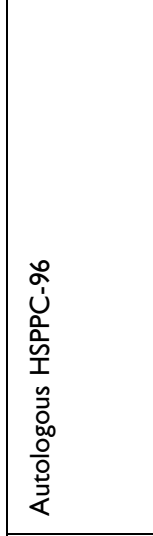 & 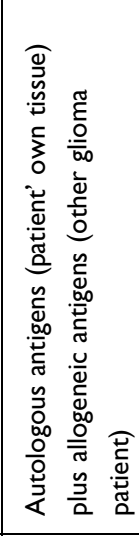 & 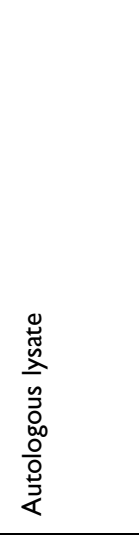 & 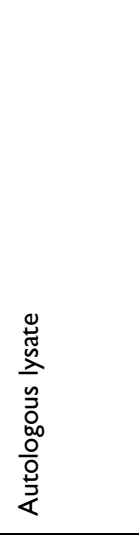 & 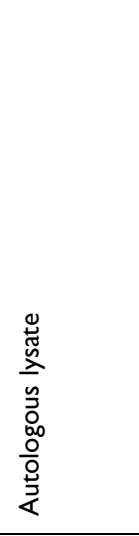 & 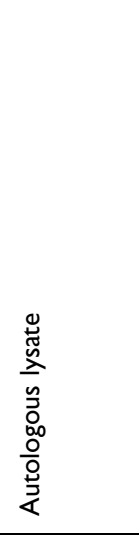 & 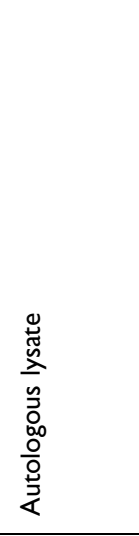 & 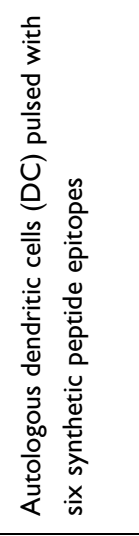 & 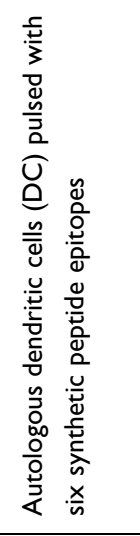 & 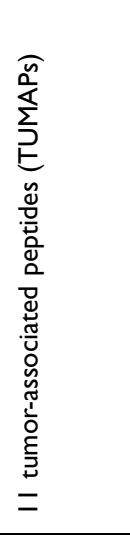 \\
\hline 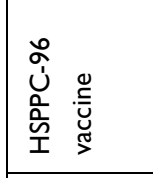 & 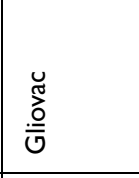 & 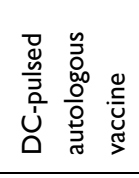 & 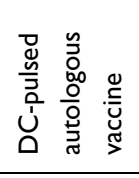 & 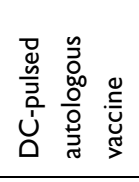 & 㳕 & 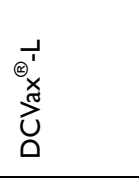 & $\underline{\underline{\hat{O}}}$ & $\begin{array}{l}\frac{\hat{o}}{1} \\
\underline{\underline{t}}\end{array}$ & 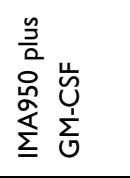 \\
\hline 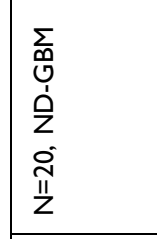 & 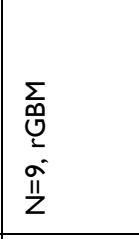 & 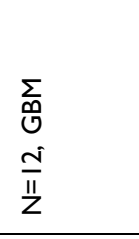 & 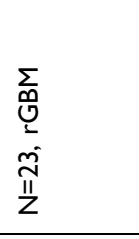 & 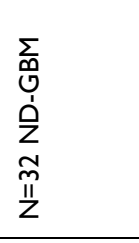 & 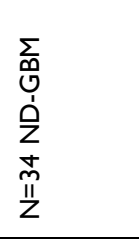 & 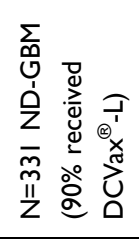 & 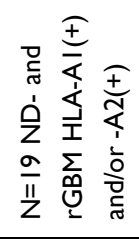 & 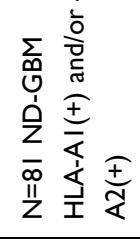 & 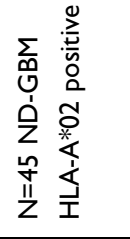 \\
\hline 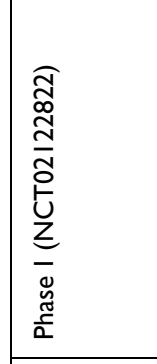 & 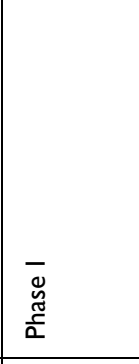 & 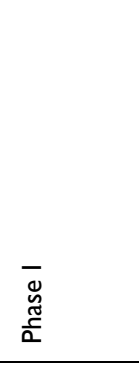 & 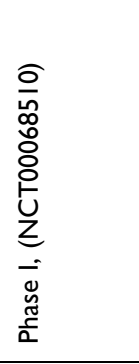 & 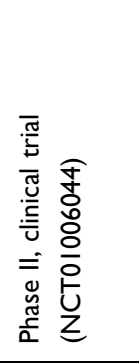 & 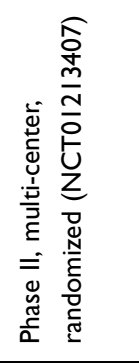 & 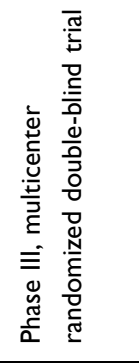 & 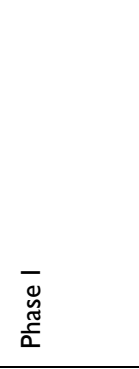 & 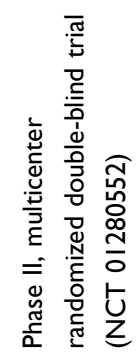 & 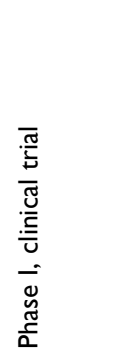 \\
\hline 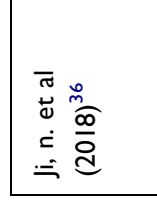 & 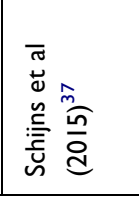 & 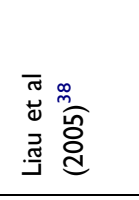 & 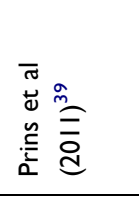 & 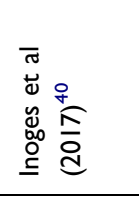 & 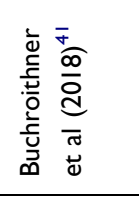 & 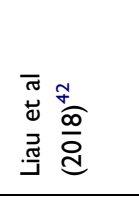 & 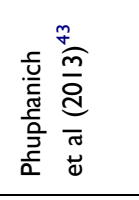 & 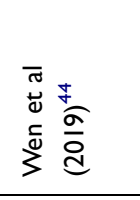 & 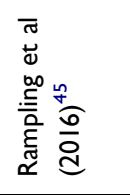 \\
\hline
\end{tabular}




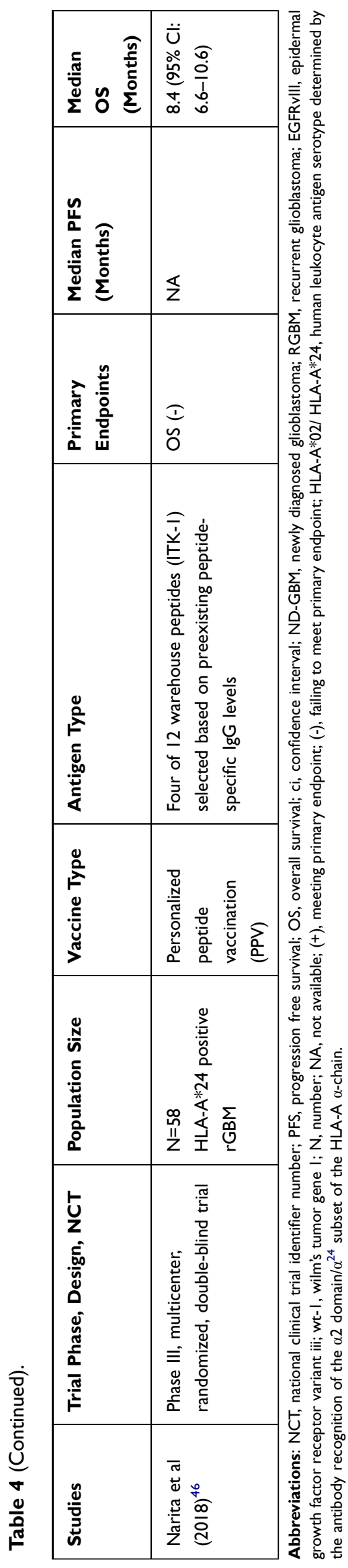

hampered immune responses in patients, as all the patients who needed it for treating side effects were not immune responders. Hence, an in-depth analysis is required to determine the optimum dose of and timing for dexamethasone administration along with chemoimmunotherapy and/ or the addition of ICIs. Furthermore, GAPVAC-101 patients received chemoradiotherapy before vaccination, whereas the participants in Keskin et al's study received only radiotherapy. In Keskin et al's trial, all patients were MGMT unmethylated, which is predictive of the clinical benefit from TMZ; hence, chemotherapy was not administered in this trial. ${ }^{53}$ GAPVAC-101 included $28.7 \%$ of the patients with hypermethylated GBM. There are several other inherent limitations with systematic reviews; for example, the data were derived from different populations and different clinical centers. Population sizes were very small; therefore, efficacy data in the form of PFS and OS should be interpreted with caution.

\section{Conclusion}

The results of these trials represent a landmark event in the vaccination paradigm for patients with GBM. Highly personalized vaccines aimed at unmutated and neoantigens have shown greater immunogenicity and safety profiles. Although survival outcomes, particularly those of the GAPVAC-101 trial, were superior to those of previous studies, further larger trials are required to be undertaken to prove treatment superiority in terms of efficacy, as observed with other vaccination strategies in this group of patients.

\section{Author Contributions}

All authors made a significant contribution to the work reported, whether that is in the conception, study design, execution, acquisition of data, analysis and interpretation, or in all these areas; took part in drafting, revising or critically reviewing the article; gave final approval of the version to be published; have agreed on the journal to which the article has been submitted; and agree to be accountable for all aspects of the work.

\section{Funding}

This work was supported by the Natural Science Foundation of Shenzhen (No. JCYJ20170307095828424), Shenzhen Health and Family Planning System Research Project (No. SZBC2017024), and the technical research and cultivation project for the youth of Shenzhen People's Hospital (No. SYKYPY2019029). 


\section{Disclosure}

We declare no conflict of interests.

\section{References}

1. Ostrom QT, Gittleman H, Xu J, et al. CBTRUS statistical report: primary brain and other central nervous system tumors diagnosed in the United States in 2009-2013. Neuro Oncol. 2016;18(suppl_5):v1v75. doi:10.1093/neuonc/now207

2. Ohgaki H, Kleihues P. The definition of primary and secondary glioblastoma. Clin Cancer Res. 2013;19(4):764-772. doi:10.1158/ 1078-0432.CCR-12-3002

3. Armstrong TS, Bishof AM, Brown PD, Klein M, Taphoorn MJB, Theodore-Oklota C. Determining priority signs and symptoms for use as clinical outcomes assessments in trials including patients with malignant gliomas: panel 1 Report. Neuro-Oncology. 2016;18 (suppl_2):ii1-ii12. doi:10.1093/neuonc/nov267

4. Sherriff J, Tamangani J, Senthil L, et al. Patterns of relapse in glioblastoma multiforme following concomitant chemoradiotherapy with temozolomide. $\mathrm{Br} \quad J$ Radiol. 2013;86(1022):20120414. doi:10.1259/bjr.20120414

5. Malmstrom A, Gronberg BH, Marosi C, et al. Temozolomide versus standard 6-week radiotherapy versus hypofractionated radiotherapy in patients older than 60 years with glioblastoma: the Nordic randomised, Phase 3 trial. Lancet Oncol. 2012;13(9):916-926. doi:10.1016/S1470-2045(12)70265-6

6. Stupp R, Mason WP, van den Bent MJ, et al. Radiotherapy plus concomitant and adjuvant temozolomide for glioblastoma. $N \mathrm{Engl}$ $J$ Med. 2005;352(10):987-996. doi:10.1056/NEJMoa043330

7. Chinot OL, Wick W, Mason W, et al. Bevacizumab plus radiotherapy-temozolomide for newly diagnosed glioblastoma. N Engl J Med. 2014;370(8):709-722. doi:10.1056/NEJMoa1308345

8. Hainsworth JD, Shih KC, Shepard GC, Tillinghast GW, Brinker BT, Spigel DR. Phase II study of concurrent radiation therapy, temozolomide, and bevacizumab followed by bevacizumab/everolimus as first-line treatment for patients with glioblastoma. Clin Adv Hematol Oncol. 2012;10(4):240-246.

9. Filley AC, Henriquez M, Dey M. Recurrent glioma clinical trial, CheckMate-143: the game is not over yet. Oncotarget. 2017;8 (53):91779-91794. doi:10.18632/oncotarget.21586

10. Winograd EK, Ciesielski MJ, Fenstermaker RA. Novel vaccines for glioblastoma: clinical update and perspective. Immunotherapy. 2016;8(11):1293-1308. doi:10.2217/imt-2016-0059

11. Aldous AR, Dong JZ. Personalized neoantigen vaccines: a new approach to cancer immunotherapy. Bioorg Med Chem. 2018;26 (10):2842-2849. doi:10.1016/j.bmc.2017.10.021

12. Li L, Goedegebuure SP, Gillanders WE. Preclinical and clinical development of neoantigen vaccines. Ann Oncol. 2017;28 (suppl_12):xii11-xii17. doi:10.1093/annonc/mdx681

13. Brown SD, Warren RL, Gibb EA, et al. Neo-antigens predicted by tumor genome meta-analysis correlate with increased patient survival. Genome Res. 2014;24(5):743-750. doi:10.1101/gr.165985.113

14. Snyder A, Makarov V, Merghoub T, et al. Genetic basis for clinical response to CTLA-4 blockade in melanoma. $N$ Engl J Med. 2014;371 (23):2189-2199. doi:10.1056/NEJMoa1406498

15. Van Allen EM, Miao D, Schilling B, et al. Genomic correlates of response to CTLA-4 blockade in metastatic melanoma. Science (New York, NY). 2015;350(6257):207-211. doi:10.1126/science.aad0095

16. Le DT, Uram JN, Wang H, et al. PD-1 blockade in tumors with mismatch-repair deficiency. New Engl $J$ Med. 2015;372 (26):2509-2520. doi:10.1056/NEJMoa1500596

17. Rizvi NA, Hellmann MD, Snyder A, et al. Cancer immunology. Mutational landscape determines sensitivity to PD-1 blockade in non-small cell lung cancer. Science (New York, NY). 2015;348 (6230):124-128. doi:10.1126/science.aaa1348
18. Hilf N, Kuttruff-Coqui S, Frenzel K, et al. Actively personalized vaccination trial for newly diagnosed glioblastoma. Nature. 2019;565(7738):240-245. doi:10.1038/s41586-018-0810-y

19. Keskin DB, Anandappa AJ, Sun J, et al. Neoantigen vaccine generates intratumoral $\mathrm{T}$ cell responses in phase $\mathrm{Ib}$ glioblastoma trial. Nature. 2019;565(7738):234-239. doi:10.1038/s41586-0180792-9

20. Moher D, Liberati A, Tetzlaff J, Altman DG. Preferred reporting items for systematic reviews and meta-analyses: the PRISMA statement. J Clin Epidemiol. 2009;62(10):1006-1012. doi:10.1016/j. jclinepi.2009.06.005

21. Higgins JPT, Altman DG, Gøtzsche PC, et al. The Cochrane Collaboration's tool for assessing risk of bias in randomised trials. BMJ. 2011;343:d5928. doi:10.1136/bmj.d5928

22. Johanns TM, Miller CA, Liu CJ, et al. Detection of neoantigen-specific $\mathrm{T}$ cells following a personalized vaccine in a patient with glioblastoma. Oncoimmunology. 2019;8(4):e1561106. doi:10.1080/2162402X.2018.1561106

23. Ridler C. Personalized vaccines use tumour fingerprint to target glioblastoma. Nat Rev Neurol. 2019;15(2):59. doi:10.1038/s41582-0190135-y

24. Cook AM, McDonnell AM, Lake RA, Nowak AK. Dexamethasone co-medication in cancer patients undergoing chemotherapy causes substantial immunomodulatory effects with implications for chemo-immunotherapy strategies. Oncoimmunology. 2016;5(3): e1066062. doi: 10.1080/2162402X.2015.1066062

25. Sampson JH, Heimberger AB, Archer GE, et al. Immunologic escape after prolonged progression-free survival with epidermal growth factor receptor variant III peptide vaccination in patients with newly diagnosed glioblastoma. J Clin Oncol. 2010;28(31):4722-4729. doi:10.1200/JCO.2010.28.6963

26. Sampson JH, Aldape KD, Archer GE, et al. Greater chemotherapy-induced lymphopenia enhances tumor-specific immune responses that eliminate EGFRvIII-expressing tumor cells in patients with glioblastoma. Neuro Oncol. 2011;13(3):324-333. doi:10.1093/ neuonc/noq157

27. Schuster J, Lai RK, Recht LD, et al. A Phase II, multicenter trial of rindopepimut (CDX-110) in newly diagnosed glioblastoma: the ACT III study. Neuro Oncol. 2015;17(6):854-861. doi:10.1093/neuonc/ nou348

28. Weller M, Butowski N, Tran DD, et al. Rindopepimut with temozolomide for patients with newly diagnosed, EGFRvIII-expressing glioblastoma (ACT IV): a randomised, double-blind, international phase 3 trial. Lancet Oncol. 2017;18(10):1373-1385. doi:10.1016/S14702045(17)30517-X

29. Izumoto S, Tsuboi A, Oka Y, et al. Phase II clinical trial of Wilms tumor 1 peptide vaccination for patients with recurrent glioblastoma multiforme. J Neurosurg. 2008;108(5):963-971. doi:10.3171/JNS/ 2008/108/5/0963

30. Hashimoto N, Tsuboi A, Kagawa N, et al. Wilms tumor 1 peptide vaccination combined with temozolomide against newly diagnosed glioblastoma: safety and impact on immunological response. Cancer Immunol, Immunother. 2015;64(6):707-716. doi:10.1007/s00262-015-1674-8

31. Oji Y, Hashimoto N, Tsuboi A, et al. Association of WT1 IgG antibody against WT1 peptide with prolonged survival in glioblastoma multiforme patients vaccinated with WT1 peptide. Int $j$ Cancer. 2016;139(6):1391-1401. doi:10.1002/ijc.30182

32. Tsuboi A, Hashimoto N, Fujiki F, et al. A phase I clinical study of a cocktail vaccine of Wilms' tumor 1 (WT1) HLA class I and II peptides for recurrent malignant glioma. Cancer Immunol, Immunother. 2019;68(2):331-340. doi:10.1007/s00262-018-2274-1

33. Fenstermaker RA, Ciesielski MJ, Qiu J, et al. Clinical study of a survivin long peptide vaccine (SurVaxM) in patients with recurrent malignant glioma. Cancer Immunol, Immunother. 2016;65 (11):1339-1352. doi:10.1007/s00262-016-1890-x 
34. Crane CA, Han SJ, Ahn B, et al. Individual patient-specific immunity against high-grade glioma after vaccination with autologous tumor derived peptides bound to the $96 \mathrm{KD}$ chaperone protein. Clin Cancer Res. 2013;19(1):205. doi:10.1158/10780432.CCR-11-3358

35. Bloch O, Lim M, Sughrue ME, et al. Autologous heat shock protein peptide vaccination for newly diagnosed glioblastoma: impact of peripheral PD-L1 expression on response to therapy. Clin Cancer Res. 2017;23(14):3575. doi:10.1158/1078-0432.CCR-16-1369

36. Ji N, Zhang Y, Liu Y, et al. Heat shock protein peptide complex-96 vaccination for newly diagnosed glioblastoma: a Phase I, single-arm trial. JCI Insight. 2018;3(10). doi:10.1172/jci.insight.99145.

37. Schijns VE, Pretto C, Devillers L, et al. First clinical results of a personalized immunotherapeutic vaccine against recurrent, incompletely resected, treatment-resistant glioblastoma multiforme (GBM) tumors, based on combined allo- and auto-immune tumor reactivity. Vaccine. 2015;33(23):2690-2696. doi:10.1016/j.vaccine.2015.03.095

38. Liau LM, Prins RM, Kiertscher SM, et al. Dendritic cell vaccination in glioblastoma patients induces systemic and intracranial T-cell responses modulated by the local central nervous system tumor microenvironment. Clin Cancer Res. 2005;11(15):5515-5525. doi:10.1158/1078-0432.CCR-05-0464

39. Prins RM, Soto H, Konkankit V, et al. Gene expression profile correlates with T-cell infiltration and relative survival in glioblastoma patients vaccinated with dendritic cell immunotherapy. Clin Cancer Res. 2011;17(6):1603-1615. doi:10.1158/1078-0432.CCR-10-2563

40. Inoges S, Tejada S, de Cerio AL, et al. A phase II trial of autologous dendritic cell vaccination and radiochemotherapy following fluorescence-guided surgery in newly diagnosed glioblastoma patients. J Transl Med. 2017;15(1):104. doi:10.1186/s12967-017-1202-z

41. Buchroithner J, Erhart F, Pichler J, et al. Audencel immunotherapy based on dendritic cells has no effect on overall and progression-free survival in newly diagnosed glioblastoma: a phase ii randomized trial. Cancers. 2018;10(10):372. doi:10.3390/cancers10100372

42. Liau LM, Ashkan K, Tran DD, et al. First results on survival from a large Phase 3 clinical trial of an autologous dendritic cell vaccine in newly diagnosed glioblastoma. J Transl Med. 2018;16(1):142. doi:10.1186/s12967-018-1507-6

43. Phuphanich S, Wheeler CJ, Rudnick JD, et al. Phase I trial of a multi-epitope-pulsed dendritic cell vaccine for patients with newly diagnosed glioblastoma. Cancer Immunol, Immunother. 2013;62 (1):125-135. doi:10.1007/s00262-012-1319-0
44. Wen PY, Reardon DA, Armstrong TS, et al. A randomized double-blind placebo-controlled Phase II trial of dendritic cell vaccine ICT-107 in newly diagnosed patients with glioblastoma. Clin Cancer Res. 2019;25:5799-5807. doi:10.1158/1078-0432.CCR-19-0261

45. Rampling R, Peoples S, Mulholland PJ, et al. A cancer research UK first time in human phase I trial of IMA950 (novel multipeptide therapeutic vaccine) in patients with newly diagnosed glioblastoma. Clin Cancer Res. 2016;22(19):4776-4785. doi:10.1158/1078-0432. CCR-16-0506

46. Narita Y, Arakawa Y, Yamasaki F, et al. A randomized, double-blind, phase III trial of personalized peptide vaccination for recurrent glioblastoma. Neuro-Oncology. 2018;21(3):348-359. doi:10.1093/ neuonc/noy 200

47. Ott PA, Hu Z, Keskin DB, et al. An immunogenic personal neoantigen vaccine for patients with melanoma. Nature. 2017;547 (7662):217-221. doi:10.1038/nature22991

48. Chu Y, Liu Q, Wei J, Liu B. Personalized cancer neoantigen vaccines come of age. Theranostics. 2018;8(15):4238-4246. doi:10.7150/ thno. 24387

49. Samstein RM, Lee C-H, Shoushtari AN, et al. Tumor mutational load predicts survival after immunotherapy across multiple cancer types. Nat Genet. 2019;51(2):202-206. doi:10.1038/s41588-018-0312-8

50. Garg AD, Vandenberk L, Van Woensel M, et al. Preclinical efficacy of immune-checkpoint monotherapy does not recapitulate corresponding biomarkers-based clinical predictions in glioblastoma. Oncoimmunology. 2017;6(4):e1295903. doi:10.1080/ 2162402X.2017.1295903

51. Giles AJ, Hutchinson M-KND, Sonnemann HM, et al. Dexamethasone-induced immunosuppression: mechanisms and implications for immunotherapy. J Immunother Cancer. 2018;6 (1):51. doi:10.1186/s40425-018-0371-5

52. Wang X, Guo G, Guan H, Yu Y, Lu J, Yu J. Challenges and potential of PD-1/PD-L1 checkpoint blockade immunotherapy for glioblastoma. J Exp Clin Cancer Res. 2019;38(1):87. doi:10.1186/ s13046-019-1085-3

53. Taylor JW, Schiff D. Treatment considerations for MGMT-unmethylated glioblastoma. Curr Neurol Neurosci Rep. 2015;15(1):507. doi:10.1007/s11910-014-0507-z
International Journal of General Medicine

\section{Publish your work in this journal}

The International Journal of General Medicine is an international, peer-reviewed open-access journal that focuses on general and internal medicine, pathogenesis, epidemiology, diagnosis, monitoring and treatment protocols. The journal is characterized by the rapid reporting of reviews, original research and clinical studies across all disease areas. The manuscript management system is completely online and includes a very quick and fair peer-review system, which is all easy to use. Visit http://www.dovepress.com/ testimonials.php to read real quotes from published authors. 